\title{
A comprehensive energy system optimization method considering variable operating condition characteristics of equipment
}

\author{
Yongli Wang ${ }^{1}$, Suhang $\mathrm{Yao}^{1, *}$, Siyi $\mathrm{Tao}^{1}$, Yuze $\mathrm{Ma}^{1}$, Yanchao $\mathrm{Lu}^{2}$ \\ ${ }^{1}$ North China Electric Power University, Beijing, 102206, China \\ ${ }^{2}$ State Grid Economic and Technological Research Institute Co., Ltd., Changping District, Beijing 102209, China
}

\begin{abstract}
The economy of the Regional Integrated Energy System (RIES) scheduling scheme is affected by the parameter accuracy of different energy conversion equipment models. The traditional static energy hub (EH) model regards the equipment efficiency as a constant and ignores the variable condition characteristics of the equipment efficiency changing with the load rate. This paper presents an optimal scheduling method of RIES considering the characteristics of equipment under variable operating conditions. Firstly, the architecture of the integrated energy system is analyzed. Secondly, based on the DEH model, an optimal scheduling method of RIES was proposed considering the characteristics of equipment under variable operating conditions. Finally, an optimal scheduling analysis is performed for a typical RIs. The results show that the proposed method can improve the accuracy of the equipment model and reduce the cost prediction error by considering the variable operating conditions of the equipment, thus providing a more economical scheduling scheme for Ries.
\end{abstract}

\section{Introduction}

The comprehensive energy system is an intelligent energy system, which improves the comprehensive utilization efficiency, sustainability and safety reliability through the deep integration and close interaction of various energy sources [1-3].Literature [4] established an Energy Hub (EH) model to describe the relationship among energy transmission, conversion, distribution and storage in RIs through a linear coupling matrix, providing a general solution for the modeling of different forms of RIs. Literature [5] proposes an optimal scheduling model for EH with energy storage equipment. Based on the EH model, literature [6-8] further considers the influence of demand-side response and uncertainty of renewable energy in optimal scheduling. However, in fact, energy conversion equipment usually has obvious nonlinear variable working condition characteristics, that is, the equipment efficiency will change with different load rates and environmental factors. [9].

Therefore, a Dynamic Energy Hub (DEH) model is established in this paper to fully consider the influence of load rate on efficiency of energy conversion equipment and improve the parameter accuracy of $\mathrm{EH}$ model. Furthermore, a DEH-based optimal scheduling method of RIES was proposed to minimize the operating cost of RIES. The influence of considering equipment load rate on the optimal scheduling scheme of RIES was analyzed through the results of typical optimal scheduling examples of RIES. The results show that this method can effectively improve the accuracy of equipment modeling, improve the economy of system operation, and make the scheduling scheme more reasonable.

\section{Integrated Energy System Framework}

The integrated energy system connects the capacity equipment, switching equipment, energy storage equipment and other equipment together through the energy supply network, and forms a unified integrated energy system together with end-user load and energy management and dispatching center. The generalized structure of the integrated energy system is shown in the figure below.

\footnotetext{
* Corresponding author: 120202206303@ncepu.edu.cn
} 


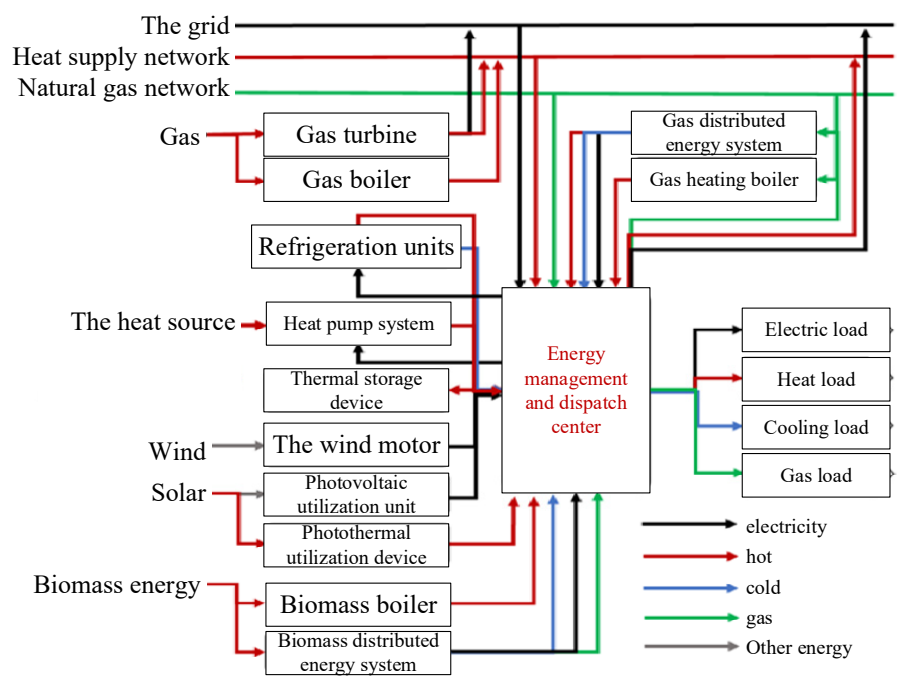

Fig. 1. Generalized structure diagram of integrated energy system

\section{RIES' mathematical model}

\subsection{DEH model}

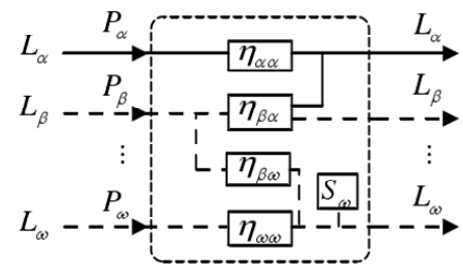

(a) Traditional EH model
The traditional EH model proposed in Literature [4] is shown in Fig. 2. A coupling matrix $\mathrm{C}$ is used to describe the functional relationship between input and output of a multi-energy system, as shown in Equation (1).

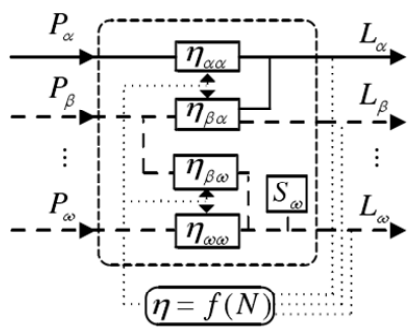

(b) Dynamic EH model

Fig. 2. Model of EH

$$
\begin{gathered}
{\left[\begin{array}{c}
L_{\alpha} \\
L_{\beta} \\
\vdots \\
L_{\omega}
\end{array}\right]=\underbrace{\left[\begin{array}{cccc}
c_{\alpha \alpha} & c_{\beta \alpha} & \cdots & c_{\omega \alpha} \\
c_{\alpha \beta} & c_{\beta \beta} & \cdots & c_{\omega \beta} \\
\vdots & \vdots & \ddots & \vdots \\
c_{\alpha \omega} & c_{\beta \omega} & \cdots & c_{\omega \omega}
\end{array}\right]}_{C}\left[\begin{array}{c}
P_{\alpha} \\
P_{\beta} \\
\vdots \\
P_{\omega}
\end{array}\right]-\underbrace{\left[\begin{array}{cccc}
s_{\alpha \alpha} & s_{\beta \alpha} & \cdots & s_{\omega \alpha} \\
s_{\alpha \beta} & s_{\beta \beta} & \cdots & s_{\omega \beta} \\
\vdots & \vdots & \ddots & \vdots \\
s_{\alpha \omega} & s_{\beta \omega} & \cdots & s_{\omega \omega}
\end{array}\right]}_{S} \underbrace{\left[\begin{array}{c}
E_{\alpha} \\
E_{\beta} \\
\vdots \\
E_{\omega}
\end{array}\right]}_{E}} \\
c_{\alpha \beta}=v_{\alpha \beta} \eta_{\alpha \beta} \quad \forall \alpha, \beta \in \psi \\
S_{\alpha}=\left\{\begin{array}{c}
\eta_{\alpha, D}, \quad \text { release energy } \\
1 / \eta_{\alpha, \mathrm{C}}, \quad \text { charge energy }
\end{array}\right.
\end{gathered}
$$

In the formula, $\alpha, \beta, \cdots, \omega$ is the element in the energy form set $\psi$, such as electricity and heat, etc. $\mathrm{L}$ is the output power vector; $\mathrm{C}$ is the coupling matrix; $\mathrm{P}$ is the input power vector; $\mathrm{S}$ is the ownership coefficient matrix of energy storage devices; $\mathrm{E}$ is the actual charging and discharging energy vector of the energy storage device.; ${ }^{\nu}{ }_{\alpha \beta}$ is the energy distribution coefficient of energy input form $\alpha$ converted to energy output form $\beta ; \eta_{\alpha \beta}$ is the efficiency of the corresponding energy conversion equipment, $\eta_{\alpha, \mathrm{C}}$ and $\eta_{\alpha, D}$ are the charging and discharging efficiency of the energy storage equipment respectively.

However, for most energy conversion devices, the energy conversion efficiency varies with the load rate.

$$
\eta=f(N)
$$

In the formula, $\eta$ is the efficiency of energy conversion equipment; $N$ is the load rate of the energy conversion equipment.

For different energy conversion equipment, the specific expression of the function relationship between the efficiency and the load rate can be obtained by polynomial fitting, which is called the efficiency model of the energy conversion equipment. The DEH model is constructed as shown in Fig. 2(b), and the DEH model as shown in Equation (5).

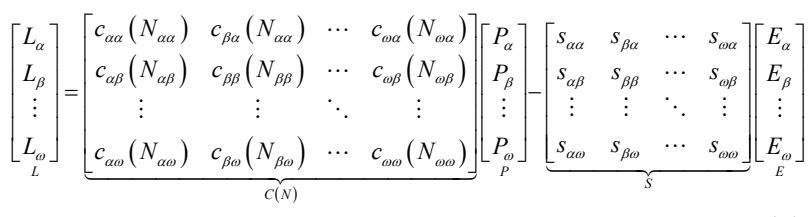




\subsection{Based on DEH's RIEs model}

\subsubsection{Variable working condition model of energy conversion equipment}

Energy conversion equipment output power constraints can be expressed as:

$$
0 \leq P_{\text {out }} \leq P_{\text {out }}^{\max }
$$

In the formula, $P_{\text {out }}^{\max }$ represents the equipment capacity, $\mathrm{kW}, P_{\text {out }}$ is the output energy of the equipment, $\mathrm{kW}$.

The relationship between energy conversion equipment efficiency and load rate can be obtained through data fitting. The relationship between some typical equipment efficiency and load rate is as follows:

1) Variable working condition model of CHP unit

$$
\eta_{C H P}=\sum_{n=0}^{4}\left(k_{C H P, \mathrm{n}} N_{C H P}^{n}\right)
$$

Where: $\eta_{C H P}$ is the electrical efficiency of CHP unit; $\mathrm{n}$ is the fitting order, $k_{C H P, \mathrm{n}}$ is the fitting coefficient of order n; $N_{C H P}$ is the electrical load rate, that is, the ratio of output electrical power to capacity.

The thermoelectric ratio of CHP unit is considered as a quadratic function of the output electrical load rate.

$$
\alpha_{C H P}=\sum_{n=0}^{2}\left(k_{\alpha, \mathrm{n}} N_{C H P}^{n}\right)
$$

Where, $\alpha_{C H P}$ is the thermoelectric ratio of CHP unit, namely the ratio of output thermal power to electric power; $k_{\alpha, \mathrm{n}}$ is the fitting coefficient of order $\mathrm{n}$.

2) Variable working condition model of gas-fired boiler

$$
\eta_{G B}=\sum_{n=0}^{1}\left(k_{\mathrm{GB}, \mathrm{n}} N_{G B}^{n}\right)
$$

Where: $\eta_{G B}$ is the efficiency of gas-fired boiler; $N_{G B}$ is the load rate of gas-fired boiler; $k_{\mathrm{GB}, \mathrm{n}}$ is the fitting coefficient of order $n$.

\subsubsection{Energy storage device model}

In this paper, it is assumed that the charging and discharging power of the energy storage device is constant in the time period of $\Delta t$, then the energy relationship stored by the device before and after charging and discharging is as follows:

$$
W_{E S, t+1}=W_{E S, t}\left(1-\sigma_{E S}+E_{E S, t} \Delta t\right)
$$

In the formula, $W_{E S, t}$ and $W_{E S, t+1}$ are the stored energy of the equipment before and after energy charging and discharging, $\mathrm{kW} \cdot \mathrm{h} ; \sigma_{E S}$ is the selfdischarging energy rate of energy storage equipment; $E_{E S, t}$ is the actual charging and discharging power of the energy storage device at time $t$ after considering the loss of charging and discharging efficiency, $\mathrm{kW} ; \Delta t$ is the optimal scheduling step size, $\mathrm{h}$.

Restrictions on the charging and discharging power of energy storage devices can be described as follows:

$$
\begin{aligned}
& 0 \leq E_{E S, t} / \eta_{E S, C} \leq P_{E S, C}^{\max } \\
& 0 \leq E_{E S, t} / \eta_{E S, D} \leq P_{E S, D}^{\max }
\end{aligned}
$$

Where, $\eta_{E S, C}$ and $\eta_{E S, \mathrm{D}}$ are energy charging and discharging efficiency of energy storage equipment respectively; $P_{E S, C}^{\max }$ and $P_{E S, \mathrm{D}}^{\max }$ are the upper limit of charging and discharging power of the equipment, $\mathrm{kW}$.

The energy storage constraint of the energy storage device is:

$$
0 \leq W_{E S, t} \leq W_{E S}^{\max }
$$

Where: ${ }_{E S}^{\max }$ is the maximum storage energy of the equipment, $\mathrm{kW} \cdot \mathrm{h} ; W_{E S, t}$ is the energy storage of the equipment at time $\mathrm{t}, \mathrm{kW} \cdot \mathrm{h}$.

\section{RIEs optimization scheduling model}

\subsection{The objective function}

This paper takes economy as the objective to optimize the scheduling of RIs. System cost A includes natural gas consumption cost $\mathrm{B}$ and grid interaction cost $\mathrm{C}$.

$$
\begin{gathered}
C=C_{g}+C_{\text {elec }} \\
C_{g}=p_{g} F_{\text {gas }} \\
C_{\text {elec }}=p_{s} \max \left(E_{\text {grid }}, 0\right)+p_{b} \min \left(E_{\text {grid }}, 0\right)
\end{gathered}
$$

In the formula, $p_{g}$ is the unit price of natural gas, $F_{\text {gas }}$ is the amount of gas purchased, $p_{s}$ and $p_{b}$ are respectively the price of electricity purchased from the power grid and the price of electricity recovered from the power grid, and $E_{\text {grid }}$ is the amount of electricity interacting with the power grid.

\subsection{The constraint}

\subsubsection{Device model constraint}

1) Constraint of energy conversion equipment

Equipment model (6) is included. Specific variable working condition characteristic models of each device are shown in Equations (7-9).

2) Energy storage device constraints 
Equipment model (10-13) are included.

It is assumed that the energy stored is consistent at the beginning and end of the scheduling cycle:

$$
W_{E S, \text { start }}=W_{E S \text {, end }}
$$

In the formula, $\mathrm{A}$ and $\mathrm{B}$ respectively represent the stored energy at the beginning and end of the equipment scheduling cycle, $\mathrm{kW} \cdot \mathrm{h}$.

\subsubsection{System operation constraint}

1) System energy balance constraint.

The energy balance constraint of the system based on $\mathrm{DEH}$ is shown in Equation (5).

2) Power constraint of the tie line.

$$
0 \leq P_{\text {elec }, t} \leq P_{\text {elec }, t}^{\max }
$$

Where, $P_{\text {elec,t }}$ is the power purchased at time period t, $P_{\text {elec }, t}^{\max }$ is the upper limit of the power of the tie line, $\mathrm{kW}$.

Aiming at the above model, this paper uses AIMMS to solve the problem.

\section{The example analysis}

\subsection{Example system and parameter selection}

In this paper, an example is selected from a park in northern China, and its system structure is shown in Figure 3. The relevant parameters of the equipment are shown in Table 1.

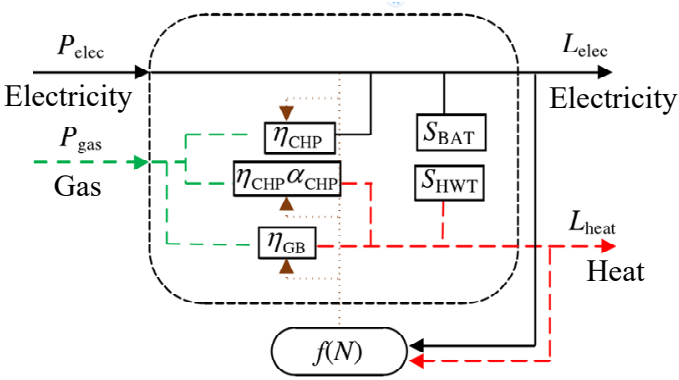

Fig. 3. Configuration of the test system

Table 1. Parameter of devices

\begin{tabular}{|c|c|c|}
\hline equipment & The parameter types & Value and unit \\
\hline \multirow{4}{*}{ CHP } & Rated capacity & $1600 \mathrm{KW}$ \\
\cline { 2 - 3 } & Rated electrical efficiency & 0.34 \\
\cline { 2 - 3 } & Rated thermoelectric ratio & 1.48 \\
\cline { 2 - 3 } & Electrical efficiency fitting coefficient & $k_{C H P, 4}=0.06, k_{C H P, 3}=-0.11, k_{C H P, 2}=-0.14, k_{C H P, 1}=0.44, k_{C H P, 0}=0.09$ \\
\cline { 2 - 3 } Gas boiler & Thermoelectric matching coefficient & $k_{C H P, 2}=3.60, k_{C H P \alpha, 1}=-5.84, k_{C H P \alpha, 0}=3.82$ \\
\cline { 2 - 3 } & Rated capacity & $2050 \mathrm{KW}$ \\
\cline { 2 - 3 } & Rated efficiency & 0.94 \\
\hline \multirow{4}{*}{ battery } & efficiency fitting coefficient & $k_{G B, 1}=0.13, k_{G B, 0}=0.81$ \\
\cline { 2 - 3 } & Rax charge/discharge power & $200 \mathrm{~kW} \cdot \mathrm{h}$ \\
\cline { 2 - 3 } & charge/discharge efficiency & $60 \mathrm{~kW}$ \\
\cline { 2 - 3 } & self discharge rate & 0.9 \\
\hline \multirow{3}{*}{$\begin{array}{c}\text { Heat storage } \\
\text { water tank }\end{array}$} & Rated capacity & 0.0001 \\
\cline { 2 - 3 } & maximum charging/heat release power & $50 \mathrm{~kW} \cdot \mathrm{h}$ \\
\cline { 2 - 3 } & charging/heat release efficiency & $12.5 \mathrm{~kW}$ \\
\cline { 2 - 3 } & self heat release rate & 0.9 \\
\hline
\end{tabular}

The actual operating electric and heating load of a typical day in the park is shown in Figure 5, and the time-of-use electricity price in this region is shown in Figure 6. The upper limit of power of the tie line is $3 \mathrm{MW}$, and the gas price is 4.16 yuan $/ \mathrm{m}^{3}$.

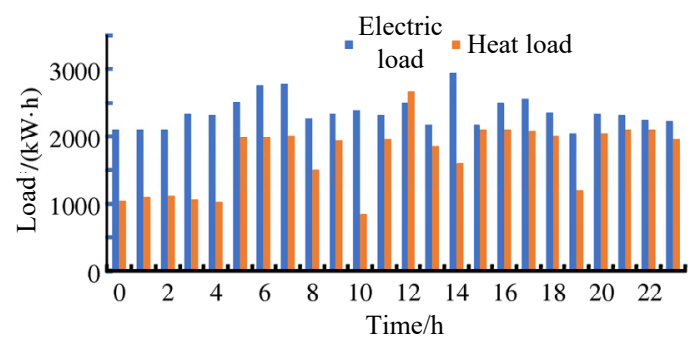

Fig. 4. Electricity and heating load

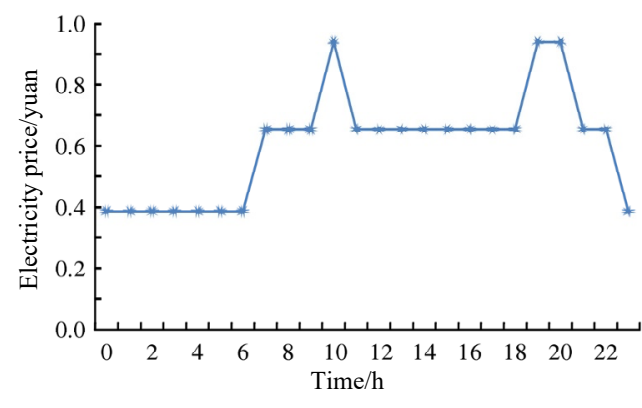

Fig.5. Time of use price

In order to verify the effectiveness of the method presented in this paper, the following two scenarios are constructed for comparison: 1) Scenario I, in which the variable working conditions of equipment are considered for optimal scheduling.2) Scenario II: Optimal scheduling is carried out without considering the 
characteristics of equipment under variable working conditions. The dispatching period is $24 \mathrm{~h}$, and the unit dispatching time is $1 \mathrm{~h}$.

\subsection{Analysis of optimal scheduling results}

The comparison results of the operating costs of RIs in one day under the two scenarios with or without considering the characteristics of equipment under varying operating conditions are shown in Table 2.

Table 2. Operation cost under two scenarios

\begin{tabular}{|c|c|c|c|c|}
\hline \multicolumn{2}{|c|}{ scenario } & $\begin{array}{c}\text { Gas } \\
\text { purchase } \\
\text { cost/yuan }\end{array}$ & $\begin{array}{c}\text { Power } \\
\text { purchase } \\
\text { cost/yuan }\end{array}$ & $\begin{array}{c}\text { Operation } \\
\text { cost/yuan }\end{array}$ \\
\hline \multirow{2}{*}{ II } & $\begin{array}{c}\text { Actual } \\
\text { Costs }\end{array}$ & 34945.60 & 21759.82 & 56705.43 \\
\cline { 2 - 6 } & $\begin{array}{c}\text { Projected } \\
\text { Costs }\end{array}$ & 33525.22 & 17327.69 & 50852.91 \\
\hline
\end{tabular}

It can be seen that the actual total operating cost of RIs in one day in Scenario I and Scenario II is RMB 52 595.03 and RMB 56705.43 respectively, and the operating cost of RIs is reduced by $7.25 \%$ after considering the characteristics of equipment under variable working conditions. Among them, the gas purchase cost in Scenario I is reduced by $41.93 \%$ compared with that in Scenario II, but the electricity purchase cost is thus increased by $48.45 \%$. It can be seen that the electricity purchase of Ries is increased in Scenario I. Electric power optimal scheduling schemes under the two scenarios are shown in Fig.6. As can be seen from Figure 6, in Scenario I, electricity is mainly purchased from the grid to meet the load demand, while in Scenario II, CHP is the main power supply.

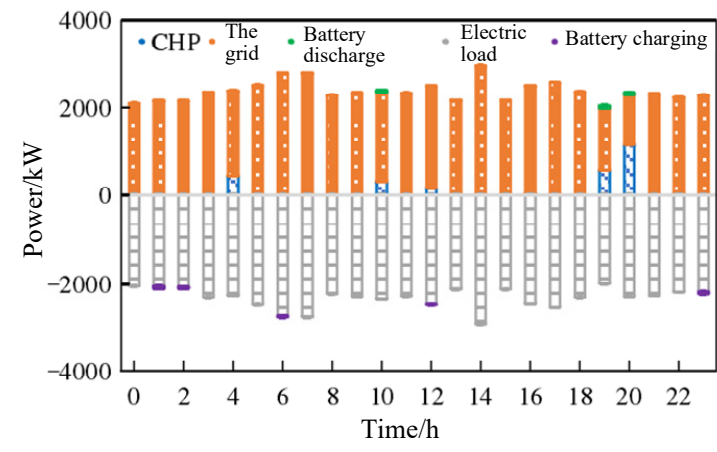

(a) Electric power optimization scheduling scheme considering variable operating conditions of equipment

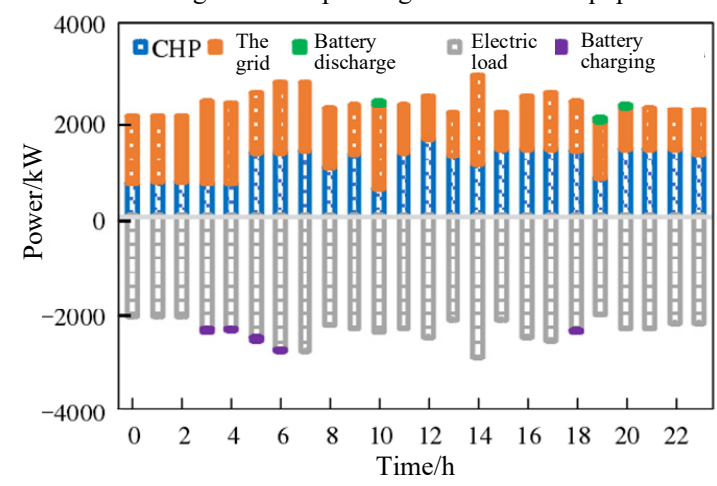

(b) Electric power optimization scheduling scheme without considering the variable operating condition characteristics of equipment

Fig. 6. Electricity load operation optimization results

In Scene I, when considering the variable working condition characteristics of equipment, the electricity purchase price from the power grid is low in the period when the electricity price is low, so most of the electric load is supplied by the power grid. At this time, the battery is charged to supplement the electrical load at the peak of the electricity price. At the peak of electricity price, the rising of electricity price increases the cost of electricity purchase from the grid, so the CHP unit starts to supply part of the electricity load. However, due to the restriction of thermal load, it cannot supply all the power load, so it still needs to purchase power from the grid. In Scenario II, under the constant efficiency model of the equipment, the charging and discharging states of the battery at different time periods are mainly affected by the electricity price, so the charging and discharging rules are basically similar to those in Scenario I. Since the equipment efficiency is regarded as the rated efficiency in Scene II, CHP has high total energy utilization rate and good economy under this condition due to the combined heat and power supply. The comparison between Scenario I and Scenario II shows that due to the adjustment of model parameters, the operation mode of the system will change significantly.

Thermal power optimization scheduling schemes under the two scenarios are shown in Fig. 7. As can be seen from the figure, gas-fired boiler is used as the main energy supply equipment for most of the time in Scene I, but CHP bears most of the heat load in Scene II, and gasfired boiler only participates in energy supply during peak energy consumption. 


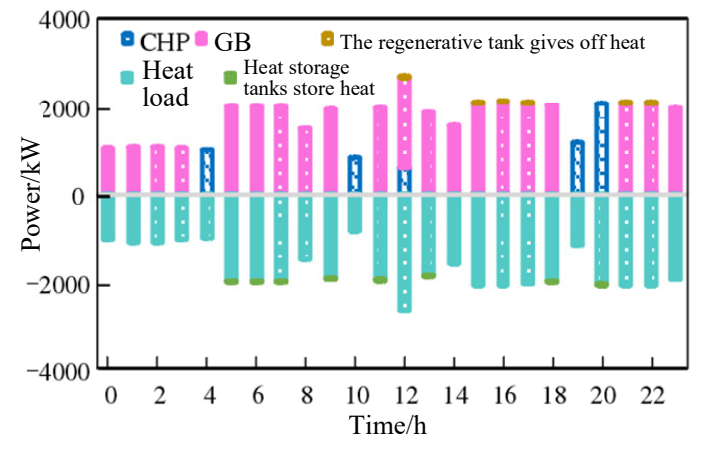

(a) Optimal scheduling scheme of thermal power considering variable operating condition characteristics of equipment

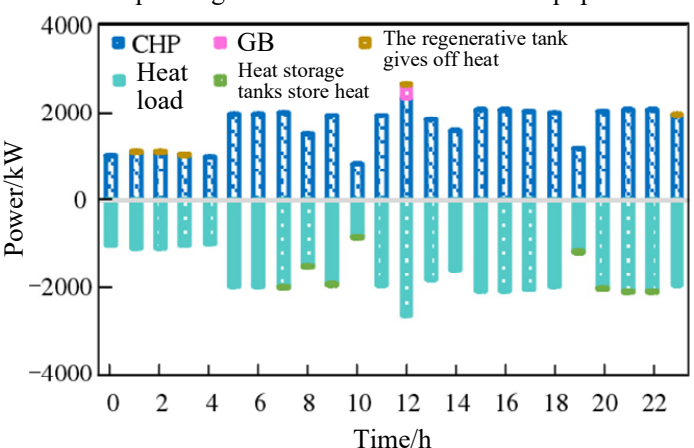

(b) Optimal scheduling scheme of thermal power without considering the characteristics of equipment under variable operating conditions

Fig. 7. Heating load operation optimization results

\section{Conclusion}

In this paper, a DEH model is constructed and an optimal scheduling method for regional comprehensive energy system is proposed considering the variable operating conditions of equipment. The conclusions are as follows:

1) Traditional EH model cannot describe the process of equipment efficiency changing with load rate. By modeling $\mathrm{DEH}$, the variable working condition characteristics of the conversion equipment can be reflected.

2) The RIES optimization scheduling method based on the variable working condition characteristics of the equipment proposed in this paper takes the variable working condition characteristics of the energy conversion equipment into consideration and improves the accuracy of the equipment model.

3) Compared with the scheduling scheme based on traditional $\mathrm{EH}$, the proposed method in this paper, considering the impact of load rate on efficiency, will ensure the operation of equipment in the high load rate range to a certain extent to improve the operation efficiency of equipment, thus saving the actual operation cost of RIEs.

\section{Acknowledgment}

The authors would like to thank the support of the project "the State Grid Corporation Science and Technology Project (SGSDDK00PDJS2000383)”.

\section{References}

1. Ma Zhao, Zhou Xiaoxin, Shang Yuwei, et al. Exploring the concept, key technologies and development model of energy internet[J]. Power System Technology, 2015, 39(11): 3014-3022(in Chinese)

2. Jia Hongjie, Mu Yunfei, Yu Xiaodan. Thought about the integrated energy system in China[J]. Electric Power Construction, 2015, 36(1): 16-25(in Chinese)

3. Zeng Ming, Liu Yingxin, Zhou Pengcheng, et al. Review and prospects of integrated energy system modeling and benefit evaluation[J]. Power System Technology, 2018, 42(6): 1697-1708(in Chinese)

4. Geidl M, Andersson G. Optimal power flow of multiple energy carriers[J]. IEEE Transactions on Power Systems, 2007, 22(1): 145-155.

5. Geidl M, Koeppel G, Favreperrod P, et al. Energy hubs for the future[J]. IEEE Power \& Energy Magazine, 2007, 5(1): 24-30.

6. Heidari A, Mortazavi S S, Bansal R C. Stochastic effects of ice storage on improvement of an energy hub optimal operation including demand response and renewable energies [J]. Applied Energy, 2020, 261(1): 114393.

7. Pazouki S, Haghifam M R R, Moser A. Uncertainty modeling in optimal operation of energy hub in presence of wind, storage and demand response[J]. International Journal of Electrical Power \& Energy Systems, 2014, 61(8): 335-345.

8. Zhang X, Shahidehpour M, Alabdulwahab A, et al. Hourly electricity demand response in the stochastic day-ahead scheduling of coordinated electricity and natural gas network[J]. IEEE Transactions on Power Systems, 2016, 31(1): 592601.

9. Wang Y, Cheng J, Zhang N, et al. Automatic and linearized modeling of energy hub and its flexibility analysis[J]. Applied Energy, 2018, 211(2): 705-714. 